\title{
Summary of a report for Canadian oral health professionals for a safe return to clinical practice during COVID-19
}

\author{
Office of the Chief Dental Officer of Canada ${ }^{1}$
}

\begin{abstract}
Following the onset of the coronavirus disease 2019 (COVID-19) pandemic, Canadian healthcare providers were advised or mandated by their regulatory bodies to cease all routine and elective care and only provide emergent/urgent care in March 2020. Two months later, the provincial/territorial governments initiated plans to "re-open" their jurisdictions; however, oral health practitioners are returning to practice in a very different environment, particularly in the domain of infection control and prevention, to the one they left prior to the onset of the pandemic. During the COVID-19 pandemic, Canadian oral health professional decision-makers at all levels have been making decisions and providing advice and guidance in a highly complex, rapidly evolving environment, often based on imperfect and/ or incomplete information. To gather, summarize and present these changes in oral health workplace environments and protocols, the Office of the Chief Dental Officer of Canada has commissioned the development of a multidisciplinary, high-level national expert review document, which resides in the public domain. This document is available for Canada's oral health regulatory authorities, educators, program officials and policy makers.
\end{abstract}

This work is licensed under a Creative Commons Attribution 4.0 International License.

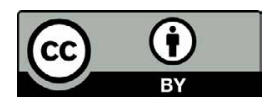

Affiliation

1 Office of the Chief Dental Officer of Canada, Public Health Agency of Canada, Ottawa, ON

\section{Correspondence:}

claudia.gorenko@canada.ca
Suggested citation: Office of the Chief Dental Officer of Canada. Summary of a report for Canadian oral health professionals for a safe return to clinical practice during COVID-19. Can Commun Dis Rep 2020;46(11/12):374-5. https://doi.org/10.14745/ccdr.v46i1112a01

Keywords: oral health, clinical practice, post-pandemic, Canada, COVID-19

\section{Introduction}

Oral health professional organizational, institutional, clinical and other leaders, as well as frontline dental professionals treating patients, are making decisions each day on how to best manage patients and to guide the professions in the context of the return to clinical practice during the coronavirus disease 2019 (COVID-19) pandemic. These people and organizations are making decisions in a very fast-moving crisis with a changing environment, with multiple, evolving sources of information and in all Canadian jurisdictions. These decisions are made based on instructions and guidelines from governments and other legal entities (such as regulatory authorities), on scientific data and evidence, and on expert opinion and on prioritized needs. They include health care, economic, ethical and other important elements, while also recognizing the information and advice upon which decisions are made is often imperfect, incomplete and/or otherwise limited. In short, oral health professional decision-makers at all levels are making decisions and providing advice and guidance in a highly complex, rapidly evolving environment, based often on imperfect and incomplete information.
A second contextual observation is that oral health practitioners (dentists, dental hygienists, dental assistants, denturists, dental technicians and dental therapists) across all jurisdictions in Canada, the vast majority of whom practice in private offices rather than in public facilities, were advised or mandated by their regulatory bodies to cease all routine and elective care and only provide emergent/urgent care in March 2020. As of May $4^{\text {th }} 2020$, a first provincial/territorial government activated a plan to "re-open" its jurisdiction, and other jurisdictions soon followed. However, oral health practitioners are returning to practice in a very different environment, particularly in the domain of infection control and prevention, to the one they left prior to the onset of the pandemic. To summarize and analyze these differences, the Office of the Chief Dental Officer of Canada (OCDOC) commissioned McGill University to draft a comprehensive document around which OCDOC then convened a representative multidisciplinary knowledge-based group from the national oral health professional and federal government health domains. A single high-level national expert consensus document on current evidence has now been generated, and 
resides in the public domain (1). Canada's oral health regulatory authorities may then choose to consult this document in developing consistent guidance for their respective registrants at the provincial/territorial level; educators, program officials and policy makers may also choose to consult this document as they carry out their respective responsibilities. Evidence gaps identified during this process have been submitted to the Canadian Institutes of Health Research by the Chief Dental Officer, with a recommendation for priority research funding consideration in these areas.

\section{Reference}

1. Office of the Chief Dental Officer. Evidence to support safe return to clinical practice by oral health professionals in Canada during the COVID-19 pandemic: A report prepared for the Office of the Chief Dental Officer of Canada. Government of Canada. www.canada.ca/en/ public-health/services/diseases/2019-novel-coronavirusinfection/health-professionals/evidence-safe-return-clinica I-practice-oral-health.html?utm_source=CCDR\&utm medium=CCDR\&utm_campaign=McGill_report_covid_ENG 